\title{
Distributed MPC-Based Path Planning for UAVs under Radio Communication Path Loss Constraints
}

\author{
Alexandra Grancharova*, Esten I. Grotli**, Tor A. Johansen** \\ * Institute of System Engineering and Robotics, Bulgarian Academy of Sciences, Acad. G. Bonchev str., Bl.2, P.O.Box 79, \\ Sofia 1113, Bulgaria (Tel: +359889625010; e-mail: alexandra.grancharova@abv.bg) \\ ** Department of Engineering Cybernetics, Norwegian University of Science and Technology, 7491 Trondheim, Norway \\ (e-mails: Esten.Ingar.Grotli@itk.ntnu.no,Tor.Arne.Johansen@itk.ntnu.no)
}

\begin{abstract}
In this paper we address the Model Predictive Control (MPC)-based path planning problem for Unmanned Aerial Vehicles (UAVs). Our goal is to find trajectories that are safe with respect to grounding and collision, fuel efficient and satisfy criteria for communication such that the UAVs form a chain with a given radio communication capacity. A centralized MPC and a distributed MPC approach to solve the path planning problem are proposed. Both approaches explicitly incorporate constraints on radio communication path losses, computed by using SPLAT!. In order to enhance the MPC problem computation, the terrain below each UAV and the communication path losses are approximated with linear functions. The control performance and the computational efficiency of the distributed MPC and the centralized MPC approaches are compared based on a simulation case study with two UAVs.
\end{abstract}

Keywords: Autonomous vehicles, Radio-communication, Path planning, Predictive control, Distributed control, Constraints.

\section{INTRODUCTION}

This research is motivated by emerging applications of UAVs (Unmanned Aerial Vehicles) such as Arctic offshore oil exploration, oil spill monitoring, and complex long range multivehicle missions in areas without a permanent communication infrastructure. The problem of path planning for UAVs has been considered in several papers. Thus, for military applications there are several systems developed for planning with respect to communication conditions, see for instance (Ukrainsky et al. (2005)). In Burdakov et al. (2009), relay chains are generated solving the all hops optimal path graph search problem. In Grotli and Johansen (2012), a Mixed-Integer Linear Programming approach for path and mission planning under radio communication constraints for UAVs is developed. Location and movement of UAVs are optimized in Han et al. (2009), in order to improve the connectivity of a wireless network. In Dixon and Frew (2007), a decentralized extremum-seeking control algorithm for nonholonomic vehicles to form a communication chain is presented. In Moses Sathyaraj et al. (2008), path planning and path finding algorithms for multiple UAVs are studied.

Model Predictive Control (MPC) is an optimization-based method for control that can handle state and input constraints (Mayne et al. (2000)). This makes the MPC methodology suitable to the optimal control of UAVs. A decentralized MPC approach to control of large scale dynamically decoupled systems (including networks of vehicles) is proposed in Keviczky et al. (2006). In particular, MPC can be used both for full control and re-planning for UAVs:

- MPC for full control: In this case there is no path planned in advance and the objective usually consists in minimizing fuel consumption, while meeting the overall mission objectives. Thus, in Dunbar and Murray (2006), a distributed MPC approach to multi-vehicle formation stabilization is proposed in which each subsystem is assigned its own optimal control problem, optimizes only for its own control at each update, and exchanges information only with neighboring subsystems. In Shim et al. (2003), a decentralized MPC method for multiple autonomous helicopters is proposed, which combines stabilization of vehicles' dynamics and trajectory generation. In Richards and How (2004) and Kuwata and How (2011), a decentralized MPC approach for path planning for cooperative UAVs in the presence of bounded disturbances is developed.

- MPC for re-planning: A path is pre-planned (i.e. first a mission planning or a path planning problem is solved), and MPC is used for re-planning to improve accuracy of the control and ensure that objectives are met despite the more inaccurate model used in the pre-planning and uncertainties that become apparent in real time. In Shin and Kim (2009), centralized and decentralized MPC methods to solve a replanning problem are compared.

In this paper, a centralized MPC and a distributed MPC approach to solve the path planning problem for UAVs are proposed, where the objective of the UAV system is to form a communication chain with a given radio communication capacity. Both approaches explicitly incorporate constraints on radio communication path losses, computed by using SPLAT! Maglicane (2010). SPLAT! is a powerful terrestrial radio frequency (RF) signal propagation and terrain analysis tool for the spectrum between $20 \mathrm{MHz}$ and $20 \mathrm{GHz}$. SPLAT! uses elevation data to calculate field strength and path loss based on the Longley-Rice Irregular Terrain Model (Longley and Rice (1968)). In order to enhance the MPC problem 
computation, the terrain below each UAV and the communication path losses are approximated with linear functions. The performance and the computational efficiency of the distributed and the centralized MPC approaches are compared based on a simulation case study with two UAVs.

\section{UAVs MODEL AND CONSTRAINTS}

\subsection{UAVs model in ENU coordinate system}

We will make use of the following coordinate systems:

ECEF: The Earth-Centered Earth-Fixed frame is a coordinate system which origin is at the center of Earth, and with axes rotating with the angular velocity of Earth.

ENU: The East-North-Up coordinate frame is a local geodetic coordinate system whose tangent plane is fitted to the geodetic reference ellipse at some convenient point for local measurements. The $x$ axis points towards east, the $y$ axis points towards true north and the $z$ axis completes the right handed orthogonal frame by pointing away from the Earth perpendicular to the reference ellipsoid.

It is assumed that $i$-th UAV is described by the following discrete time model in the ENU coordinate system:

$$
p_{i}(t+1)=I_{3} p_{i}(t)+T_{\mathrm{S}} I_{3} v_{i}(t),
$$

$i=1, \ldots, n_{\mathrm{UAV}}$, where $n_{\mathrm{UAV}}$ is the number of UAVs, and $p_{i}(t)=\left[x_{i}(t), y_{i}(t), z_{i}(t)\right], \quad$ and $\quad v_{i}(t)=\left[v_{i x}(t), v_{i y}(t), v_{i z}(t)\right]$, with $x_{i}, y_{i}, z_{i}$ and $v_{i x}, v_{i y}, v_{i z}$ being the positions and the velocities along the orthogonal axes in a local ENU coordinate reference frame, and $T_{\mathrm{S}}$ is the sampling period. In an MPC problem formulation, the optimization is performed over velocity sequences $V_{i}=\left[v_{i, t}, v_{i, t+1}, \ldots, v_{i, t+N-1}\right]$, $i=1, \ldots, n_{\mathrm{UAV}}$ along a prediction horizon with length $N$. The predicted position $p_{i, t+k \mid t}$ of $i$-th UAV, given the initial position $p_{i, t \mid t}=p_{i}(t)$ and the velocity sequence $V_{i}$ is:

$$
p_{i, t+k+1 \mid t}=I_{3} p_{i, t+k \mid t}+T_{\mathrm{S}} I_{3} v_{i, t+k}
$$

\subsection{Constraints}

The following constraints are imposed on the UAVs.

\section{Constraints on the velocity vectors}

Assume that the minimal and the maximal allowed speeds for the UAVs are $\underline{S}_{i}, \bar{S}_{i}, i=1, \ldots, n_{\mathrm{UAV}}$. Here, the requirement for the speeds of fixed wing UAVs to be above the minimal allowed values is treated as a soft constraint, which is met by introducing an appropriate term in the cost function (see Section 3). The upper bound on $i$-th UAV's speed is respected by imposing the following hard constraints on the elements $v_{i x}, v_{i y}, v_{i z}$ of the velocity vector $v_{i}$ :

$$
\begin{aligned}
& -\bar{v}_{i} \leq v_{i x, t+k} \leq \bar{v}_{i},-\bar{v}_{i} \leq v_{i y, t+k} \leq \bar{v}_{i},-\bar{v}_{i} \leq v_{i z, t+k} \leq \bar{v}_{i} \\
& k=0,1, \ldots, N-1
\end{aligned}
$$

where $\bar{v}_{i}=\sqrt{\bar{S}_{i}^{2} / 3}$.

\section{Acceleration constraints}

The constraints on the $i$-th UAV's acceleration are:

$$
\begin{aligned}
& -\Delta_{i} \mathbf{1} \leq\left(v_{i, t}-v_{i}(t-1)\right) / T_{\mathrm{S}} \leq \Delta_{i} \mathbf{1} \\
& -\Delta_{i} \mathbf{1} \leq\left(v_{i, t+k+1}-v_{i, t+k}\right) / T_{\mathrm{S}} \leq \Delta_{i} \mathbf{1}, k=0,1, \ldots, N-2
\end{aligned}
$$

where $\mathbf{1}=[1,1,1]^{T}$ and $\Delta_{i}, i=1, \ldots, n_{\mathrm{UAV}}$ are known constants.

\section{Constraints on position}

The following constraints are imposed on the position of $i$-th UAV in the ENU coordinate system:

$$
\begin{aligned}
& x_{i} \leq x_{i, t+k \mid t} \leq \bar{x}_{i}, \underline{y}_{i} \leq y_{i, t+k \mid t} \leq \bar{y}_{i}, k=1, \ldots, N \\
& \hat{h}_{i}^{\text {terr }}\left(p_{i, t+k \mid t}\right)+\delta_{h} \leq z_{i, t+k \mid t} \leq \bar{z}_{i}+r_{i}, k=1, \ldots, N
\end{aligned}
$$

where $x_{i, t+k \mid t}, y_{i, t+k \mid t}, z_{i, t+k \mid t}$ are the predicted coordinates of the $i$-th UAV by using the model (2) for a given velocity sequence $V_{i}, \underline{x}_{i}, \underline{y}_{i}$ and $\bar{x}_{i}, \bar{y}_{i}, \bar{z}_{i}$ are lower and upper bounds, $\hat{h}_{i}^{\text {terr }}\left(p_{i, t+k \mid t}\right)$ represents the approximate height of the terrain below the $i$-th UAV and within its maximal reach distance for the specified time horizon, and $\delta_{h}$ is a safety distance from ground. Here, $r_{i} \geq 0$ is a slack variable, which is introduced in order to avoid infeasibility. Collision avoidance is ensured by carefully choosing $\underline{x}_{i}, \underline{y}_{i}, \bar{x}_{i}, \bar{y}_{i}$ such that the designated area for each vehicle is non-overlapping. Furthermore, the constraints imposed on the coordinate $z$ are such that the anti-grounding requirement is met.

The height of the terrain below the $i$-th UAV is upper approximated with the following linear function:

$$
\begin{aligned}
\hat{h}_{i}^{\text {terr }}\left(p_{i, t+k \mid t}\right)= & a_{i, 1}\left(x_{i, t \mid t}, y_{i, t \mid t}\right) x_{i, t+k \mid t}+ \\
& a_{i, 2}\left(x_{i, t \mid t}, y_{i, t \mid t}\right) y_{i, t+k \mid t}+a_{i, 3}\left(x_{i, t \mid t}, y_{i, t \mid t}\right)
\end{aligned}
$$

Here, $a_{i, 1}, a_{i, 2}, a_{i, 3}$ are coefficients, which depend on the initial position of the $i$-th UAV and they are obtained by solving the Linear Programming (LP) problem:

$$
\begin{aligned}
& \min _{a_{i, 1}, a_{i, 2}, a_{i, 3}}\left(a_{i, 1} x_{i}^{\text {center }}+a_{i, 2} y_{i}^{\text {center }}+a_{i, 3}\right) \\
& \text { subject to } A_{i}\left[\begin{array}{lll}
a_{i, 1} & a_{i, 2} & a_{i, 3}
\end{array}\right]^{T} \geq h_{i}^{\text {terr }}
\end{aligned}
$$

Here, the matrix $A_{i}$ contains the $x$ and $y$ coordinates of the terrain points, which are within the maximal reach distance for the $i$-th $\mathrm{UAV}$, i.e. $A_{i}=\left[x_{i, j}^{\text {terr }} y_{i, j}^{\text {terr }} 1\right], j=1, \ldots, N_{i}$, the vector $h_{i}^{\text {terr }}=\left[z_{i, j}^{\text {terr }}\right], j=1, \ldots, N_{i}$ contains their heights, and $x_{i}^{\text {center }}, y_{i}^{\text {center }}$ are the mean $x$ and $y$ coordinates of the terrain points. An example of terrain approximation with a linear function is shown in Fig. 1.

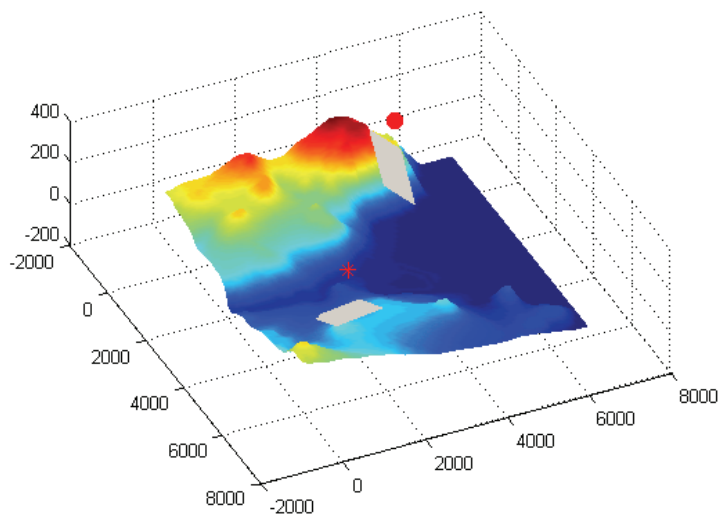

Fig. 1. Terrain approximation (grey color) below two UAVs (red circle and red star). 


\section{Constraints on radio communication path losses}

The connectivity constraints represent constraints on the path losses in the communication chain from the base station to the target station, where the topology of the chain is preassigned. The path losses are computed by using SPLAT! (Maglicane (2010)) and they are approximated by linear functions at each time sample. We use the Windows version of SPLAT!, provided by McMellen (2010). SPLAT! produces reports, graphs, and high resolution topographic maps that depict line-of-sight paths, and regional path loss and signal strength contours through which expected coverage areas of transmitters and repeater systems can be obtained. The following constraints are imposed:

- Constraint on radio communication path loss from the base station to the 1-st UAV:

$$
\hat{c}_{\text {base }-1, t+k \mid t}\left(p_{1, t+k \mid t}\right) \leq \bar{c}_{\text {base }-1}+q_{0}, k=1, \ldots, N
$$

where $\hat{c}_{\text {base- } 1, t+k \mid t}\left(p_{1, t+k \mid t}\right)$ represents the approximate path loss in the communication from the base station to the 1-st $\mathrm{UAV}, \bar{c}_{\text {base-1 }}$ is the maximal allowed path loss, and $q_{0} \geq 0$ is a slack variable. The path loss is upper approximated with the following linear function:

$$
\begin{aligned}
\hat{c}_{\text {base }-1, t+k \mid t}\left(p_{1, t+k \mid t}\right)= & b_{1,1}\left(p_{1, t \mid t}\right) x_{1, t+k \mid t}+b_{1,2}\left(p_{1, t \mid t}\right) y_{1, t+k \mid t} \\
& +b_{1,3}\left(p_{1, t \mid t}\right) z_{1, t+k \mid t}+b_{1,4}\left(p_{1, t \mid t}\right)
\end{aligned}
$$

Here, $b_{1, j}, j=1, \ldots, 4$ are coefficients, which depend on the initial position of the 1-st UAV. They are obtained by solving the following LP problem:

$$
\begin{aligned}
& \min _{b_{1,1}, b_{1,2}, b_{1,3}, b_{1,4}}\left(b_{1,1} x_{1}^{\text {center }}+b_{1,2} y_{1}^{\text {center }}+b_{1,3} z_{1}^{\text {center }}+b_{1,4}\right) \\
& \text { subject to } B_{1}\left[\begin{array}{llll}
b_{1,1} & b_{1,2} & b_{1,3} & b_{1,4}
\end{array}\right]^{T} \geq c_{\text {base- }}
\end{aligned}
$$

Here, the matrix $B_{1}=\left[\begin{array}{llll}x_{1, j} & y_{1, j} & z_{1, j} & 1\end{array}\right], j=1, \ldots, \tilde{N}_{1}$ contains the coordinates of a given number of points, which are within the maximal reach distance for the 1-st UAV, the vector $c_{\text {base- } 1}=\left[c_{\text {base- } 1, j}\right], j=1, \ldots, \tilde{N}_{1}$ contains the path losses between these points and the base station (computed with SPLAT!), and $x_{1}^{\text {center }}, y_{1}^{\text {center }}, z_{1}^{\text {center }}$ are the mean coordinates of these points.

- Constraint on radio communication path loss from $i$ th UAV to $(\boldsymbol{i}+\mathbf{1})$-th $\mathrm{UAV}, i=1, \ldots, n_{\mathrm{UAV}}-1$ :

$$
\begin{aligned}
& \hat{c}_{i-(i+1), t+k \mid t}\left(p_{i, t+k \mid t}, p_{i+1, t+k \mid t}\right) \leq \bar{c}_{i-(i+1)}+q_{i} \\
& k=1, \ldots, N, i=1, \ldots, n_{\mathrm{UAV}}-1
\end{aligned}
$$

where $\quad \hat{c}_{i-(i+1), t+k \mid t}\left(p_{i, t+k \mid t}, p_{i+1, t+k \mid t}\right) \quad$ represents the approximate path loss in the communication from $i$-th UAV to $(i+1)$-th UAV, $\bar{c}_{i-(i+1)}$ is the maximal allowed path loss, and $q_{i} \geq 0$ is a slack variable. This path loss is upper approximated with the following linear function:

$$
\begin{aligned}
& \hat{c}_{i-(i+1), t+k \mid t}\left(p_{i, t+k \mid t}, p_{i+1, t+k \mid t}\right)= \\
& b_{i+1,1}\left(p_{i, t \mid t}, p_{i+1, t \mid t}\right) x_{i, t+k \mid t}+b_{i+1,2}\left(p_{i, t \mid t}, p_{i+1, t \mid t}\right) y_{i, t+k \mid t} \\
& +b_{i+1,3}\left(p_{i, t \mid t}, p_{i+1, t \mid t}\right) z_{i, t+k \mid t}+b_{i+1,4}\left(p_{i, t \mid t}, p_{i+1, t \mid t}\right) x_{i+1, t+k \mid t} \\
& +b_{i+1,5}\left(p_{i, t \mid t}, p_{i+1, t \mid t}\right) y_{i+1, t+k \mid t}+b_{i+1,6}\left(p_{i, t \mid t}, p_{i+1, t \mid t}\right) z_{i+1, t+k \mid t} \\
& +b_{i+1,7}\left(p_{i, t \mid t}, p_{i+1, t \mid t}\right)
\end{aligned}
$$

Here, $b_{i+1, j}, j=1, \ldots, 7$ are coefficients, which depend on the initial positions of the $i$-th and $(i+1)$-th UAVs and they are obtained by solving an LP problem that is similar to the LP problem (11)-(12).

- Constraint on radio communication path loss from $n_{\mathrm{UAV}}$-th UAV to the target station:

$\hat{c}_{n_{\mathrm{UAV}}-\text { target }, t+k \mid t}\left(p_{n_{\mathrm{UAV}}, t+k \mid t}\right) \leq \bar{c}_{n_{\mathrm{UAV}} \text {-target }}+q_{n_{\mathrm{UAV}}}, k=1, \ldots, N(15)$ where $\hat{c}_{n_{\mathrm{UAV}}-\text { target }, t+k \mid t}\left(p_{n_{\mathrm{UAV}}, t+k \mid t}\right)$ is the approximate path loss in the communication from $n_{\mathrm{UAV}}$-th UAV to the target station, $\bar{c}_{n_{\mathrm{UAV}}-\text { target }}$ is the maximal allowed path loss, and $q_{n_{\mathrm{UAV}}} \geq 0$ is a slack variable. The path loss is upper approximated with the following linear function:

$$
\begin{aligned}
& \hat{c}_{n_{\mathrm{UAV}}-\operatorname{target}, t+k \mid t}\left(p_{n_{\mathrm{UAV}}, t+k \mid t}\right)= \\
& b_{n_{\mathrm{UAV}}+1,1}\left(p_{n_{\mathrm{UAV}}, t \mid t}\right) x_{n_{\mathrm{UAV}}, t+k \mid t}+b_{n_{\mathrm{UAV}}+1,2}\left(p_{n_{\mathrm{UAV}}, t \mid t}\right) y_{n_{\mathrm{UAV}}, t+k \mid t} \\
& +b_{n_{\mathrm{UAV}}+1,3}\left(p_{n_{\mathrm{UAV}}, t \mid t}\right) z_{n_{\mathrm{UAV}}, t+k \mid t}+b_{n_{\mathrm{UAV}}+1,4}\left(p_{n_{\mathrm{UAV}}, t \mid t}\right)
\end{aligned}
$$

Here, $b_{n_{\mathrm{UAV}}+1, j}, j=1, \ldots, 4$ are coefficients, which depend on the initial position of $n_{\mathrm{UAV}}$-th UAV. They are obtained by solving an LP problem, similar to above.

The purpose of approximating the radio communication path losses is to pre-compute them off-line as piecewise linear functions of the positions of UAVs by using SPLAT!. This can lead to a more computationally efficient on-line solution of the MPC-based path planning problem, where the communication path losses will be estimated simply by function evaluation.

\section{FORMULATION OF MPC PROBLEM FOR UAVs}

\subsection{Centralized MPC problem}

Consider the optimal path planning problem where the goal is to transmit the signal from the base station to the target station by using $n_{\mathrm{UAV}}$ relaying nodes (UAVs). For the current positions $p_{i}(t), i=1, \ldots, n_{\mathrm{UAV}}$ of the UAVs, the MPC solves the following centralized optimization problem:

\section{Centralized MPC:}

$J^{\mathrm{c}^{*}}=$

$$
\min _{\substack{V_{i}, i=1, \ldots, n_{\mathrm{UAV}} \\ q_{i}, i=0,1, \ldots, n_{\mathrm{UAV}} \\ r_{i}, i=1, \ldots, n_{\mathrm{UAV}}}} J^{c}\left(V_{1}, \ldots, V_{n_{\mathrm{UAV}}}, q_{0}, q_{1}, \ldots, q_{n_{\mathrm{UAV}}}, r_{1}, \ldots, r_{n_{\mathrm{UAV}}}\right)
$$

subject to $p_{i, t \mid t}=p_{i}(t), i=1, \ldots, n_{\mathrm{UAV}}$, constraints (2)-(6) for $i=1, \ldots, n_{\mathrm{UAV}}$, constraints (9), (10), (13)-(16), and:

$$
\begin{aligned}
& q_{i} \geq 0, i=0,1, \ldots, n_{\mathrm{UAV}} \\
& r_{i} \geq 0, i=1, \ldots, n_{\mathrm{UAV}}
\end{aligned}
$$

The cost function to be minimized is the following:

$$
\begin{aligned}
& J^{\mathrm{c}}\left(V_{1}, \ldots, V_{n_{\mathrm{UAV}}}, q_{0}, q_{1}, \ldots, q_{n_{\mathrm{UAV}}}, r_{1}, \ldots, r_{n_{\mathrm{UAV}}}\right)= \\
& \sum_{i=1}^{n_{\mathrm{UAV}}} \sum_{k=0}^{N-1}\left[\alpha_{i, 1} \frac{\left(S_{i, t+k}-\tilde{S}_{i}\right)^{2}}{\left(\tilde{S}_{i}-\underline{S}_{i}\right)^{2}}+\alpha_{i, 2} w_{i, t+k}^{2}\right]+\beta_{0} \frac{q_{0}^{2}}{\Delta c_{\mathrm{base}-1}^{2}}+ \\
& \sum_{i=1}^{n_{\mathrm{UAV}}-1} \beta_{i} \frac{q_{i}^{2}}{\Delta c_{i-(i+1)}^{2}}+\beta_{n_{\mathrm{UAV}}} \frac{q_{n_{\mathrm{UAV}}}^{2}}{\Delta c_{n_{\mathrm{UAV}}-\text { target }}^{2}}+\sum_{i=1}^{n_{\mathrm{UAV}}} \gamma_{i} \frac{r_{i}^{2}}{\Delta h_{i}^{2}}
\end{aligned}
$$


where $N$ is the prediction horizon, $S_{i, t+k}=\sqrt{v_{i x, t+k}^{2}+v_{i y, t+k}^{2}+v_{i z, t+k}^{2}}$ and $\tilde{S}_{i}, \quad i=1, \ldots, n_{\mathrm{UAV}}$ are the predicted speeds and the typical cruise speeds of the UAVs, $\Delta c_{i-(i+1)}$ is a constant denoting the range of change of the path loss between the $i$-th and $(i+1)$-th UAV ( $\Delta c_{\text {base-1 }}$ and $\Delta c_{n_{\mathrm{UAV}}-\text { target }}$ are defined in a similar way), $\Delta h_{i}$ is a constant denoting the range of change of the height of $i$-th $\mathrm{UAV}$, and $\alpha_{i, 1}, \alpha_{i, 2}, i=1, \ldots, n_{\mathrm{UAV}}, \beta_{i}, i=0,1, \ldots, n_{\mathrm{UAV}}$, and $\gamma_{i}, i=1, \ldots, n_{\mathrm{UAV}}$ are weighting coefficients. In (20), the terms $\left(S_{i, t+k}-\tilde{S}_{i}\right)^{2} /\left(\tilde{S}_{i}-\underline{S}_{i}\right)^{2}, \quad i=1, \ldots, n_{\mathrm{UAV}}$ reflect the requirement to minimize the deviation of the speeds of UAVs from their typical cruise speeds, while the terms $w_{i, t+k}^{2}$, $i=1, \ldots, n_{\mathrm{UAV}}$ represent soft lower bound constraints on the speeds of the UAVs. The variable $w_{i, t+k}$ is defined as:

$$
w_{i, t+k}=\left\{\begin{array}{l}
0, \text { if } S_{i, t+k} \geq \underline{S}_{i} \\
\left(\underline{S}_{i}-S_{i, t+k}\right) /\left(\tilde{S}_{i}-\underline{S}_{i}\right), \text { if } S_{i, t+k}<\underline{S}_{i}
\end{array}\right.
$$

The last terms in (20) are introduced in order to minimize the slack variables $q_{i} \geq 0, i=0,1, \ldots, n_{\mathrm{UAV}}$ (which participate in the communication path losses constraints (9), (13) and (15)) and $r_{i} \geq 0, i=1, \ldots, n_{\mathrm{UAV}}$ (which participate in the position constraints (5)).

\subsection{Distributed MPC problem}

For the current positions $p_{i}(t), i=1, \ldots, n_{\mathrm{UAV}}$ of the UAVs, the MPC solves a distributed optimization problem consisting of the following sub-problems:

MPC sub-problem for the 1-st UAV:

$$
J^{1^{*}}=\min _{V_{1}, q_{0}, q_{1}, r_{1}} J^{1}\left(V_{1}, q_{0}, q_{1}, r_{1}\right)
$$

subject to $p_{1, t \mid t}=p_{1}(t)$, constraints (2)-(6) with $i=1$, and:

$$
\begin{aligned}
& \hat{c}_{\text {base }-1, t+k \mid t}\left(p_{1, t+k \mid t}\right) \leq \bar{c}_{\text {base-1 }}+q_{0}, k=1, \ldots, N \\
& \hat{c}_{1-2, t+k \mid t}\left(p_{1, t+k \mid t}, p_{2, t \mid t}\right) \leq \bar{c}_{1-2}+q_{1} \\
& k=1, \ldots, N \\
& q_{0} \geq 0, q_{1} \geq 0, r_{1} \geq 0
\end{aligned}
$$

with the cost function defined by:

$$
\begin{aligned}
J^{1}\left(V_{1}, q_{0}, q_{1}, r_{1}\right)= & \sum_{k=0}^{N-1}\left[\alpha_{1,1} \frac{\left(S_{1, t+k}-\tilde{S}_{1}\right)^{2}}{\left(\tilde{S}_{1}-\underline{S}_{1}\right)^{2}}+\alpha_{1,2} w_{1, t+k}^{2}\right]+ \\
& \beta_{0} \frac{q_{0}^{2}}{\Delta c_{\text {base- }}^{2}}+\beta_{1} \frac{q_{1}^{2}}{\Delta c_{1-2}^{2}}+\gamma_{1} \frac{r_{1}^{2}}{\Delta h_{1}^{2}}
\end{aligned}
$$

Due to the distributed formulation, the approximated path loss $\hat{c}_{1-2, t+k \mid t}\left(p_{1, t+k \mid t}, p_{2, t \mid t}\right)$ is computed as dependent on the predicted coordinates of the 1-st UAV only, i.e.:

$$
\begin{aligned}
& \hat{c}_{1-2, t+k \mid t}\left(p_{1, t+k \mid t}, p_{2, t \mid t}\right)=b_{2,1}\left(p_{1, t \mid t}, p_{2, t \mid t}\right) x_{1, t+k \mid t} \\
& +b_{2,2}\left(p_{1, t \mid t}, p_{2, t \mid t}\right) y_{1, t+k \mid t}+b_{2,3}\left(p_{1, t \mid t}, p_{2, t \mid t}\right) z_{1, t+k \mid t} \\
& +b_{2,4}\left(p_{1, t \mid t}, p_{2, t \mid t}\right) x_{2, t \mid t}+b_{2,5}\left(p_{1, t \mid t}, p_{2, t \mid t}\right) y_{2, t \mid t} \\
& +b_{2,6}\left(p_{1, t \mid t}, p_{2, t \mid t}\right) z_{2, t \mid t}+b_{2,7}\left(p_{1, t \mid t}, p_{2, t \mid t}\right)
\end{aligned}
$$

It is assumed the 2-nd UAV communicates its current coordinates $x_{2, t \mid t}, y_{2, t \mid t}, z_{2, t \mid t}$ to the 1-st UAV.
MPC sub-problem for the $i$-th $\mathbf{U A V}, i=2, \ldots, n_{\mathrm{UAV}}-1$ :

$$
J^{i^{*}}=\min _{V_{i}, q_{i}, r_{i}} J^{i}\left(V_{i}, q_{i}, r_{i}\right)
$$

subject to $p_{i, t \mid t}=p_{i}(t)$, constraints (2)-(6), and:

$$
\begin{aligned}
& \hat{c}_{i-(i+1), t+k \mid t}\left(p_{i, t+k \mid t}, p_{i+1, t \mid t}\right) \leq \bar{c}_{i-(i+1)}+q_{i} \\
& k=1, \ldots, N \\
& q_{i} \geq 0, r_{i} \geq 0
\end{aligned}
$$

with the cost function defined by:

$$
\begin{gathered}
J^{i}\left(V_{i}, q_{i}, r_{i}\right)=\sum_{k=0}^{N-1}\left[\alpha_{i, 1} \frac{\left(S_{i, t+k}-\tilde{S}_{i}\right)^{2}}{\left(\tilde{S}_{i}-\underline{S}_{i}\right)^{2}}+\alpha_{i, 2} w_{i, t+k}^{2}\right]+ \\
\beta_{i} \frac{q_{i}^{2}}{\Delta c_{i-(i+1)}^{2}}+\gamma_{i} \frac{r_{i}^{2}}{\Delta h_{i}^{2}}
\end{gathered}
$$

Similar to above, the approximated path loss $\hat{c}_{i-(i+1), t+k \mid t}\left(p_{i, t+k \mid t}, p_{i+1, t \mid t}\right)$ is computed as dependent on the predicted coordinates only of the $i$-th $\operatorname{UAV}\left(x_{i, t+k \mid t}, y_{i, t+k \mid t}\right.$, $\left.z_{i, t+k \mid t}\right)$. The (i+1)-th UAV communicates its current coordinates $x_{i+1, t \mid t}, y_{i+1, t \mid t}, z_{i+1, t \mid t}$ to the $i$-th UAV.

MPC sub-problem for the $n_{\mathrm{UAV}}$-th UAV:

$$
J^{n_{\mathrm{UAV}}{ }^{*}}=\min _{V_{n_{\mathrm{UAV}}}, q_{n_{\mathrm{UAV}}}, r_{n_{\mathrm{UAV}}}} J^{n_{\mathrm{UAV}}}\left(V_{n_{\mathrm{UAV}}}, q_{n_{\mathrm{UAV}}}, r_{n_{\mathrm{UAV}}}\right)
$$

subject to $p_{n_{\mathrm{UAV}}, t \mid t}=p_{n_{\mathrm{UAV}}}(t)$, constraints (2)-(6) with $i=n_{\mathrm{UAV}}$, and:

$$
\begin{aligned}
& \hat{c}_{n_{\mathrm{UAV}}-\text { target }, t+k \mid t}\left(p_{n_{\mathrm{UAV}}, t+k \mid t}\right) \leq \bar{c}_{n_{\mathrm{UAV}}-\text { target }}+q_{n_{\mathrm{UAV}}} \\
& k=1, \ldots, N \\
& q_{n_{\mathrm{UAV}}} \geq 0, r_{n_{\mathrm{UAV}}} \geq 0
\end{aligned}
$$

with the cost function defined by:

$$
\begin{aligned}
& J^{n_{\mathrm{UAV}}}\left(V_{n_{\mathrm{UAV}}}, q_{n_{\mathrm{UAV}}}, r_{n_{\mathrm{UAV}}}\right)= \\
& \sum_{k=0}^{N-1}\left[\alpha_{n_{\mathrm{UAV}}, 1} \frac{\left(S_{n_{\mathrm{UAV}}, t+k}-\tilde{S}_{n_{\mathrm{UAV}}}\right)^{2}}{\left(\tilde{S}_{n_{\mathrm{UAV}}}-\underline{S}_{n_{\mathrm{UAV}}}\right)^{2}}+\alpha_{n_{\mathrm{UAV}}, 2} w_{n_{\mathrm{UAV}}, t+k}^{2}\right]+ \\
& \beta_{n_{\mathrm{UAV}}} \frac{q_{n_{\mathrm{UAV}}}^{2}}{\Delta c_{n_{\mathrm{UAV}}-\text { target }}^{2}}+\gamma_{n_{\mathrm{UAV}}} \frac{r_{n_{\mathrm{UAV}}}^{2}}{\Delta h_{n_{\mathrm{UAV}}}^{2}}
\end{aligned}
$$

\section{SIMULATION RESULTS}

In this section we use two UAVs to form a communication chain from a base station to a moving target. The origin of the ENU frame is at longitude, latitude and height, specified by the vector $X^{\mathrm{WGS}}=(63.400,10.32,0)$. The base station is located at $(6000,1000,150)$. The initial position of the target is $(100,6000,10)$ and its speed is $8[\mathrm{~m} / \mathrm{s}]$ towards east. The initial positions of the UAVs are at $(5000,3000,300)$ and $(3800,3800,300)$. The parameter values for the two UAVs, as well as for the optimization problem are given in Table 1.

The distributed MPC and the centralized MPC approaches, described in the previous section, are compared and the results are presented in Fig. 2 to Fig. 7. The initial positions of the two UAVs and the target are marked with yellow, while their final positions are marked with blue. 
Table 1. Parameter values.

\begin{tabular}{|cc|}
\hline Parameter & Value \\
\hline$\underline{x_{1}}, \bar{x}_{1}, \underline{y}_{1}, \bar{y}_{1}, \bar{z}_{1}$ & $4100,7000,0,3500,100,600$ \\
\hline$\underline{x}_{2}, \bar{x}_{2}, \underline{y}_{2}, \bar{y}_{2}, \bar{z}_{2}$ & $0,3900,3500,5800,100,600$ \\
\hline$\delta_{h}, \Delta h_{1}, \Delta h_{2}$ & $100[\mathrm{~m}], 800[\mathrm{~m}], 500[\mathrm{~m}]$ \\
\hline$\underline{S}_{1}, \underline{S}_{2}, \bar{S}_{1}, \bar{S}_{2}$ & $6[\mathrm{~m} / \mathrm{s}], 6[\mathrm{~m} / \mathrm{s}], 33[\mathrm{~m} / \mathrm{s}], 25[\mathrm{~m} / \mathrm{s}]$ \\
\hline$\tilde{S}_{1}, \tilde{S}_{2}$ & $15[\mathrm{~m} / \mathrm{s}], 11[\mathrm{~m} / \mathrm{s}]$ \\
\hline$\Delta_{1}, \Delta_{2}$ & $1\left[\mathrm{~m} / \mathrm{s}^{2}\right], 1\left[\mathrm{~m} / \mathrm{s}^{2}\right]$ \\
\hline $\bar{c}_{\text {base-1 }}, \bar{c}_{1-2}, \bar{c}_{2-\text { target }}$ & $108[\mathrm{~dB}], 112[\mathrm{~dB}], 108[\mathrm{~dB}]$ \\
\hline$\Delta c_{\text {base-1 }}, \Delta c_{1-2}, \Delta c_{2-\text { target }}$ & $7[\mathrm{~dB}], 10[\mathrm{~dB}], 20[\mathrm{~dB}]$ \\
\hline$T_{\mathrm{S}}, N$ & $1[\mathrm{~s}], 10$ \\
\hline$\alpha_{1,1}, \alpha_{2,1}, \alpha_{1,2}, \alpha_{2,2}$ & $1,1,1,1$ \\
\hline$\beta_{0}, \beta_{1}, \beta_{2}, \gamma_{1}, \gamma_{2}$ & $1,1,1,1,1$ \\
\hline
\end{tabular}
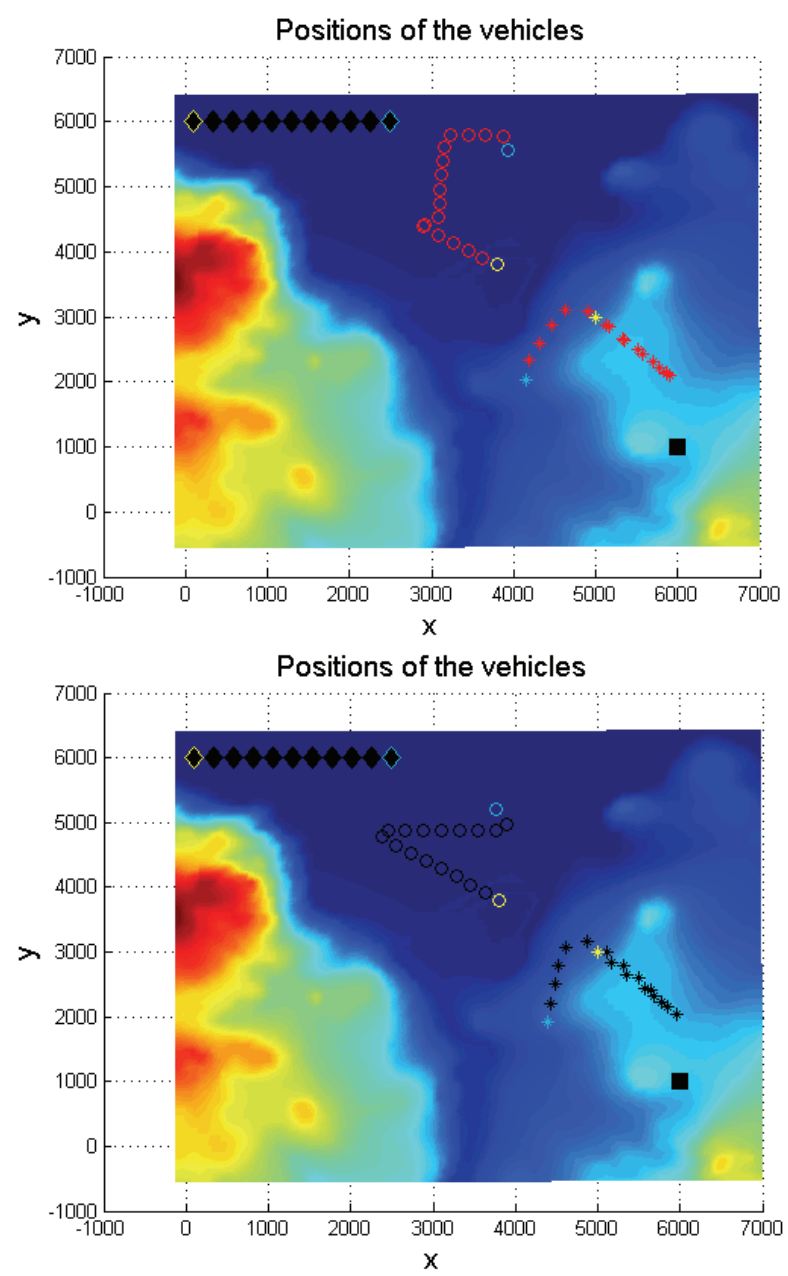

Fig. 2. Positions of UAVs (1-st UAV - star, 2-nd UAV circle), the base station (square), the moving target (diamond). Top: Position of UAVs with the distributed MPC. Bottom: Position of UAVs with the centralized MPC.

It can be seen that both MPC approaches produce feasible trajectories. From Fig. 3 it can be observed that the two UAVs need to change their heights in order to satisfy all constraints in the optimization problem. The non-smoothness of the path loss curve in Fig. 7 is due to the fact that the position of the moving target is updated every 30 [s].
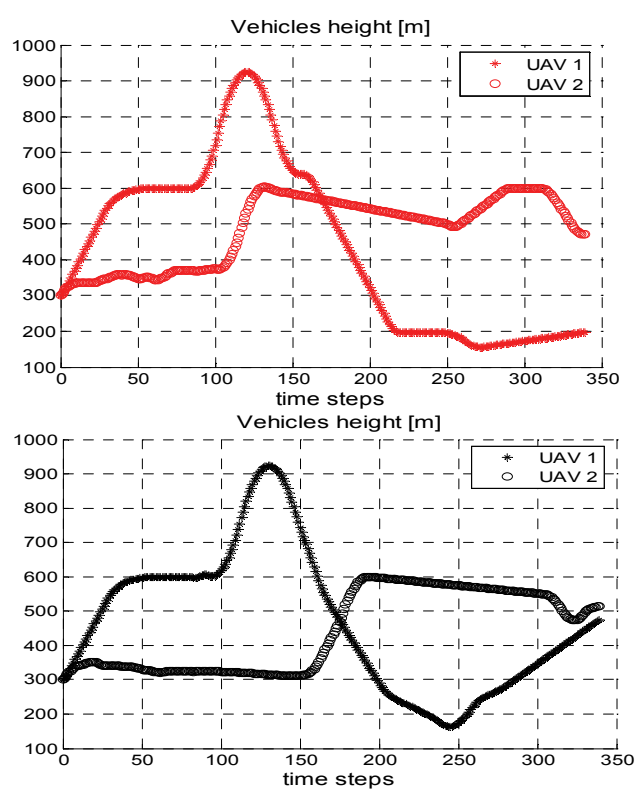

Fig. 3. Heights of UAVs with the distributed MPC (red curves) and with the centralized MPC (black curves).

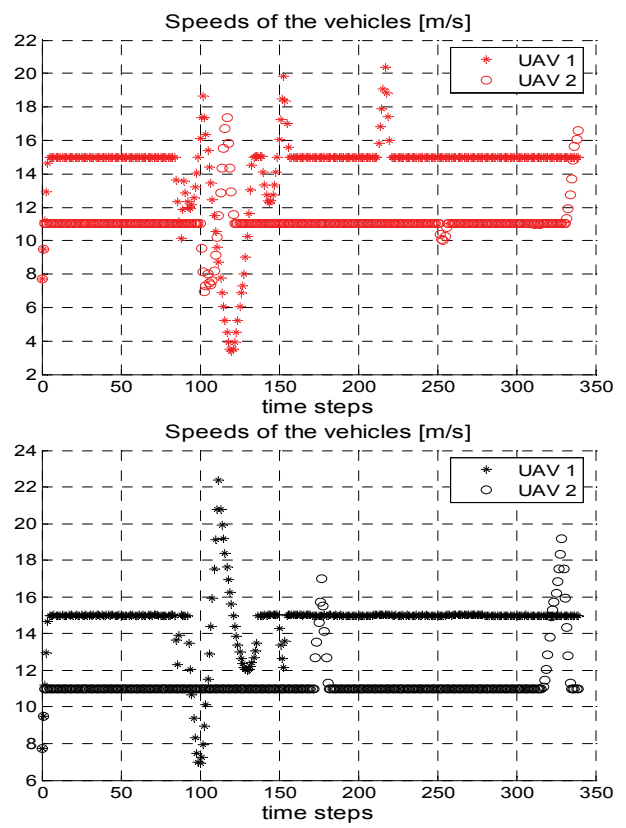

Fig. 4. Speeds of UAVs with the distributed MPC (red curves) and with the centralized MPC (black curves).

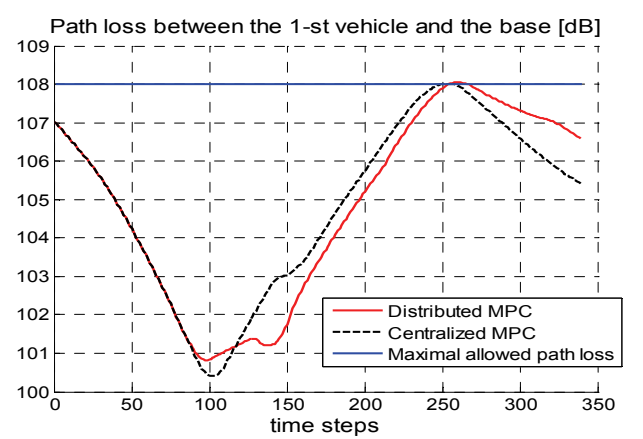

Fig. 5. Radio communication path loss between the 1-st UAV and the base station. 


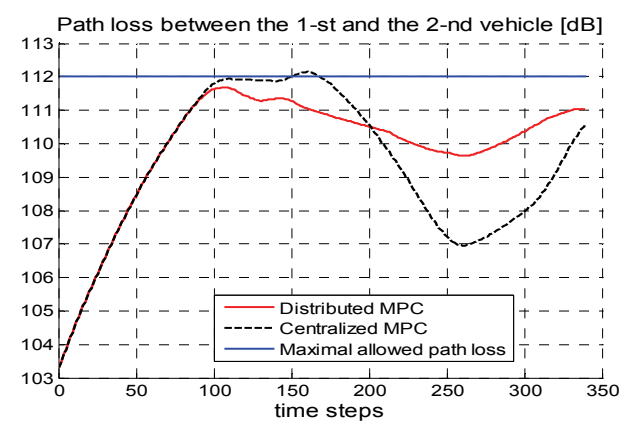

Fig. 6. Radio communication path loss between the 1-st UAV and the 2-nd UAV.

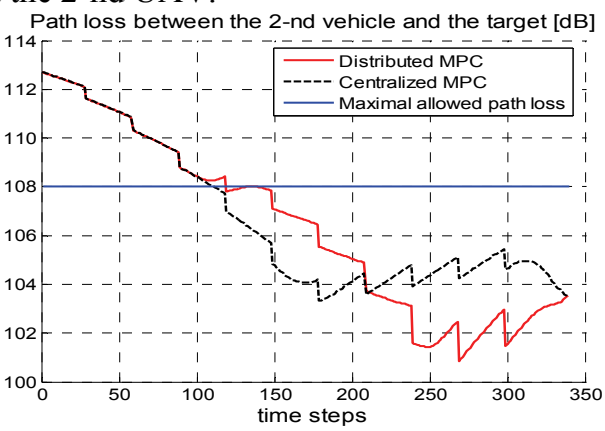

Fig. 7. Radio communication path loss between the 2-nd UAV and the target.

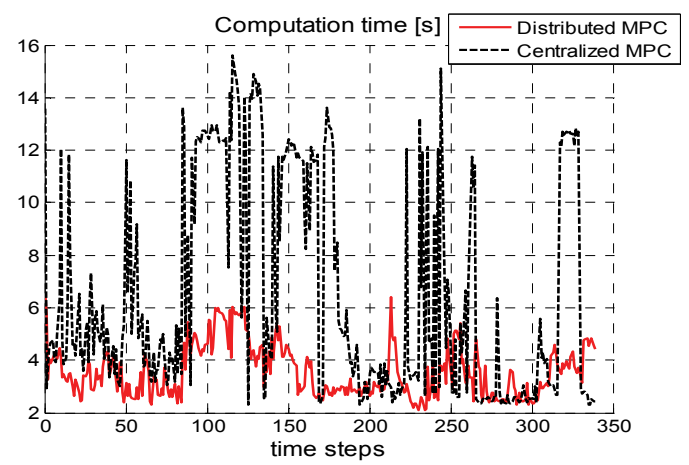

Fig. 8. CPU time.

The computation of the distributed MPC and the centralized MPC is performed on a $3 \mathrm{GHz}$ Intel Core 2 Duo processor with Matlab implementation. The associated CPU times are given in Fig. 8. It can be seen that the distributed MPC requires significantly less computational efforts in comparison to the centralized MPC. It should be noted that due to the nonlinearity of the cost functions (20), (26), (31), (35), a nonlinear programming solver is used to solve the distributed MPC and the centralized MPC problems. These cost functions are discontinuous, but convex and therefore their global minimum can be found for the linearized constraints.

However, due to the linearization used with the proposed distributed MPC approach, it may not find globally optimal trajectories in complicated terrains that would lead to nonconvex constraints if no linearization was made. The approach therefore has limitations compared to global approaches. Since the distributed MPC approach is more towards optimizing trajectories than to stabilize motion at given trajectories, the focus has been on optimality rather than stability analysis.

\section{REFERENCES}

Burdakov, B., Doherty, P., Holmberg, K., Kvarnström, J., and Olsson, P.-M. (2009). Positioning unmanned aerial vehicles as communication relays for surveillance tasks. Robotics Science and Systems, Online Proceedings.

Dixon, C. and Frew, E.W. (2007). Decentralized extremumseeking control of nonholonomic vehicles to form a communication chain. In Advances in Cooperative Control and Optimization, Springer, pp. 311-322.

Dunbar, W.B. and Murray, R.M. (2006). Distributed receding horizon control for multi-vehicle formation stabilization. Automatica, 42, 549-558.

Grøtli, E.I. and Johansen, T.A. (2012). Path planning for UAVs under communication constraints using SPLAT! and MILP. Journal of Intelligent and Robotic Systems, 65, 265-282.

Han, Z., Swindlehurst, A., and Liu, K. (2009). Optimization of manet connectivity via smart deployment/movement of unmanned air vehicles. IEEE Transactions on Vehicular Technology, 58, 3533-3546.

Keviczky, T., Borrelli, F., and Balas, G.J. (2006). Decentralized receding horizon control for large scale dynamically decoupled systems. Automatica, 42, 21052115.

Kuwata, Y. and How, J.P. (2011). Cooperative distributed robust trajectory optimization using receding horizon MILP. IEEE Transactions on Control Systems Technology, 19, 423-431.

Longley, A.G. and Rice, P.L. (1968). Prediction of tropospheric radio transmission loss over irregular terrain: A computer method. U. S. Goverment, Tech. Rep.

Maglicane, J. (2010). SPLAT! An RF signal propagation, loss and terrain analysis tool. http://www.qsl.net/kd2bd/splat.html.

Mayne, D.Q., Rawlings, J.B., Rao, C.V., and Scokaert, P.O.M. (2000). Constrained model predictive control: Stability and optimality. Automatica, 36, 789-814.

McMellen, J. (2010). RF propagation modeling with SPLAT! for Windows. http://blog.gearz.net/2007/09/rfpropagation-modeling-with-splat-for.html.

Moses Sathyaraj, B., Jain, L.C., Finn, A., and Drake, S. (2008). Multiple UAVs path planning algorithms: a comparative study. Fuzzy Optimization and Decision Making, 7, 257-267.

Richards, A. and How, J.P. (2004). Decentralized model predictive control of cooperating UAVs. In Proc. of IEEE Conference on Decision and Control, pp. 4286-4291.

Shim, D.H., Kim, H.J., and Sastry, S. (2003). Decentralized nonlinear model predictive control of multiple flying robots. In Proc. of IEEE Conference on Decision and Control, Maui, Hawaii USA, pp. 3621-3626.

Shin, J. and Kim, H.J. (2009). Nonlinear model predictive formation flight. IEEE Transactions on Systems, Man, and Cybernetics-Part A: Systems and Humans, 39, 11161125.

Ukrainsky, O., Zebrowitz, H., Hein, C., Cortese, A., Rubin, A., Poon, C., Bard, A., and Reyes, H. (2005). An open environment for rapid embedded planning of on-the-move communications networks using multi-level abstraction. In Proc. of Military Communications Conference. 